\title{
Bemerkungen zu einer Geschichte der französischen Heldensage.
}

Von Carl Voretzsch, der sich an der Erforschung des französischen Heldenepos schon mehrfach in hervorragender Weise beteiligt hat, liegt seit kurzem ein stattlicher Band vor, der über Komposition und Quellen des "Huon von Bordeaux" handelt. Der Verfasser eröffnet damit eine Sammlung, die er „Epische Studien: Beiträge zur Geschichte der frz. Heldensage und Heldendichtung" betitelt hat. Sein letztes Ziel ist eine „Geschichte der frz. Heldensage", oder, da nach seiner Anschauung dic Ependichter ihren Stoff vornehmlich aus der Sage geschöpft haben, eine „Stoffgeschichte des frz. Heldenepos". Das Programm dieses Werkes hat er schon in der Tübinger Antrittsvorlesung vom Jahr 1894 entworfen. Nun gedenkt er durch ausführliche Untersuchungen lehrreicher Einzelprobleme die geplante Gesamtdarstellung vorzubereiten. Diesem Zweck sollen die „Epischen Studien“ dienen. Angesichts der hohen und schönen Aufgabe, die sich der Verfasser gestellt hat, dürfte jetzt die Gelegenheit sein, eine Reihe Fragen zur Sprache zu bringen, die im Rahmen einer "Geschichte der frz. Heldensage" berücksichtigt zu werden verdienen. Voretzsch hat, wie er ausdrücklich bemerkt, eine "Stoffgeschichte“ im Auge; darauf will er seine Aufgabe beschränken. Will man aber feststellen, was jeder Dichter aus seinem Stoffe gemacht, warum er eben diesen und keinen andern gewählt und warum er denselben so oder so abgeändert hat, dann lälst sich die Frage nach dem Thema des Dichters nicht umgehen. Bei der Bestimmung seines Themas pflegt sich jeder Künstler gemeiniglich nach seinem Publikum zu richten. Manche Gelehrte haben auf diese und andere Dinge, die von entscheidender Wichtigkeit sind, bisher weniger geachtet. Deshalb sollen diese Fragen hier im Zusammenhang besprochen werden. Teilweise habe ich darüber schon gehandelt in Vollmöllers Jahresbericht, wo ich in dem Abschnitt "Germanisches in der altfranzösischen Dichtung $1891-96$ " über die älteren Arbeiten des Verfassers referiert habe. Auf diesen Aufsatz (Vollmöller IV. Bd., 2. Teil, S. 416-26) sei hier von vornherein verwiesen.

I. Am lebhaftesten erörtert wurde in den letzten Jahren die Frage nach den Quellen der Ependichter. Haben wir als

Zeitechr. f. rom. Phil. XXV. 
Zwischenglied zwischen den geschichtlichen Ereignissen und den Epen lyrisch-epische Lieder anzusetzen, oder Sagen d. h. Volksüberlieferungen, oder die Berichte der Historiographen? Dazu sind neuerdings zwei weitere Anschauungen vermittelnder Natur gekommen. Voretzsch widmet vorzugsweise diesen letzteren einen einleitenden Teil seines Buches, betitelt „Kritische Bemerkungen über Begriff und Bedeutung der Sage".

a) Einige der grolsen Heldenepen bei Indern, Griechen, Germanen, Franzosen, Finnen und Karakirgisen, erwiesen sich, als man ihre Komposition genauer prüfte, zum Teil aus kleineren ursprünglich selbständigen Dichtungen zusammengestellt. Hier steht am Anfang Friedrich August Wolfs berühmte Homerkritik. Zuerst Lachmann machte daraus ein System, als er die Methode Wolfs an Nibelungen und Ilias durchführte und die ursprünglichen Einzellieder im Wortlaut wiederherzustellen versuchte. Von Lachmann stammt die sogenannte "Liedertheorie". Sie wurde von G. Paris auf die altfrz. Epen angewendet und bis heute mit Entschiedenheit vertreten: cantilenae, chants lyrico-épiques, chants contemporains, als eine Art Romanzen gedacht, bildeten nach ihm die notwendige Vorstufe der Epen. Ich habe schon in Vollmöllers Jahresbericht (S. 421) darauf hingewiesen, dafs der Gedanke, Epen aus lyrischen Liedern hervorgehen $\mathrm{zu}$ lassen, in einer bestimmten ästhetischen Doktrin wurzelt. Es war im letzten Grunde Herders Lehre von der Priorität der Lyrik. 1

b) Zuerst bei den Germanisten regte sich der Widerspruch gegen die Liedertheorie. Zwar erkannte man die Fälle an, wo sich einzelne Kompositionsteile in der That als ursprünglich selbständige Dichtungen erweisen liefsen. Aber man sträubte sich gegen die dogmatische Durchführung dieses Gedankens. Zwischen den ältesten Gedichten überhaupt und dem geschichtlichen Ereignis setzte man als Mittelglied mündliche Volksüberlieferungen ein, die "Sage“ im engeren Sinn des Wortes. ${ }^{2}$ Unter anderen vertrat auch Uhland diesen Standpunkt. Schon früher gab A.W. Schlegel seine klassische Begriffsbestimmung der Sage, in seiner berühmten Recension der altdeutschen Wälder der Brüder Grimm, vom Jahre 1815. Er sagte hier: ${ }^{3}$ "Die ältesten Heldenlieder haben fast immer eine geschichtliche Grundlage oder wenigstens Veranlassung, und diese war aus der Sage geschöpft. Unter der Sage verstehen wir das Andenken merkwürdiger Begebenheiten, wie es sich von einem Geschlecht und zuweilen von einem Volk zum andern fortpflanzt. ... Vorliebe oder Abneigung, dann der dem menschlichen Geist besonders in der ersten Frische der Ein-

1 Vgl. Herders "Lyra" (Terpsichore 2. Teil) in Suphan-Redlich XXVII (Poet. Werke III), Berlin 1881, S. 179. Bezeichnenderweise eröffnet L. Gautier seine Epopées fr. mit dieser These (I 2, S. 4-5).

2 Ueber den verschiedenen Wortsinn der "Sage" vgl. mein Referat im Jahresbericht S. 417.

${ }^{3}$ ed. Böcking XII, S. $3^{87}$. 
bildungskraft inwohnende Hang zum Wunderbaren, brachten Uebertreibungen hervor, und die Ruhmbegierde falste sie willig auf. Wer hätte nicht gern vernommen, wer hätte bezweifeln mögen, dals das kriegerische Volk, zu dem er gehörte, von einem übernatürlichen Heldengeschlecht abstamme? ... Aus obigen Umständen erhellet, wie die Sage, noch ehe sie dichterisch behandelt wurde, schon in gewissem Grade den Forderungen der Poesie entsprach, so dafs der Dichter nur kühnlich in derselben Richtung fortzugehn brauchte." Bei den Romanisten wies nach Uhland P. Meyer nachdrücklich auf die Bedeutung der Sage für die Entstehung des Heldenepos hin. Doch blieb bis heute die Liedertheorie hier in ungeschwächtem Ansehen, obwohl $\mathrm{H}$. Suchier und mit besonderem Nachdruck auch Voretzsch die Berechtigung dieser Lehre angezweifelt hatten. $\mathrm{Da}$ ist es denn von Interesse, dafs Voretzsch im vorliegenden Bande zeigt, wie G. Paris sowohl als L. Gautier früher neben den Liedern auch die tradition orale = legende als Quelle der Ependichter angenommen haben (S. 3-I I).

c) Diesen beiden Theorieen hat es, wenigstens in ihrer älteren Formulierung, nicht zum Vorteil gereicht, dafs sie mehr auf dem Boden kunstphilosophischer Lehren als aus der Praxis des Litterarhistorikers erwachsen waren. Wolfs und seiner Nachfolger Liedertheorie stützte sich, wie bemerkt, auf die Ueberzeugung vom höheren Alter der Lyrik. Die Brüder Grimm und die meisten Romantiker, so auch Ludwig Uhland, standen der Lehre Schellings nahe, dafs die Poesie in ihrer Entwicklung vom unbewursten Schaffen ausgehe und erst später bewurst erzeugt werde. Auch Hegels Konzeption des objektiven Volksgeistes wirkte malsgebend ein. Die Hegelsche Schule, so Fr. Th. Vischer, trug den Satz vor, dafs die älteste Poësie, Sage, Märchen und Mythus, nicht von Individualitäten, sondern vom Volksgeist selber unbewufst geschaffen sei. Schliefslich machte sich eine Zeitlang der Einflufs von Steinthals Völkerpsychologie geltend. Man schrieb der Sage sogar eine Art Selbstthätigkeit zu, indem man vom „Walten der Sage“ und ähnlichem sprach. Auch Voretzsch hat sich, wohl unter der Einwirkung Uhlands, wenigstens im Ausdruck von solchen Vorstellungen nicht ganz frei gehalten; in meinem Referat (S. 417-418) habe ich meine Einwendungen dagegen geltend gemacht. Es wäre ein wichtiger Beitrag zur Geschichte der neueren Philologie, wenn man diesen Einflüssen metaphysischer Spekulation auf die Litteraturgeschichte im einzelnen nachgehen wollte. Gemeinsam war allen diesen Anschauungen, gleichviel ob sie sich an Schelling, Hegel oder Steinthal anlehnten, und ob sie sich in der Liedertheorie oder in der Sagentheorie bemerklich machten, der eine grundsätzliche Irrtum, dafs man der künstlerischen Individualität, durch die allein ein ästhetisch Wertvolles erzeugt wird, ihren Platz bestritt. Zuerst hat kein geringerer als A. W. Schlegel seine Stimme dagegen erhoben. Er schrieb:1 „Die Sage und volksmälsige Dich-

1 ed. Böcking XII, S. 385. 
tung war allerdings das Gesamteigentum der Zeiten und Völker, aber nicht eben so ihre gemeinsame Hervorbringung. Was man an Zeitaltern und Völkern rühmt, löset sïch immer bei näherer Betrachtung in die Eigenschaften und Handlungen einzelner Menschen auf; und soll man hiebei der Anhäufung und Wiederholung des Gemeinen, oder dem seltenen Auftreten des Aufserordentlichen den grölsten Einflufs zuschreiben? ... Die Steine sind nicht der Thurm: diesen schuf der Entwurf des Baumeisters." Trotz diesen klaren und deutlichen Ausführungen Schlegels drang die Vorstellung von einem „unbewufst schaffenden Volksdichter" in viele litterarhistorische Darstellungen auch der neueren Zeit ein. Ich habe meinerseits zu zeigen versucht (Vollmöller IV, 2, S. 418), dafs die Sage zwar Eigentum des Volks, aber das Werk dichterisch begabter Persönlichkeiten innerhalb desselben ist.

Aus dem begreiflichen Widerstreben gegen Voraussetzungen wie die geschilderten glaube ich es verstehen zu können, dals $\mathrm{Ph}$. Aug. Becker die Lieder sowohl als die Sagen rundweg ablehnte und geschriebene Geschichtswerke als einzige Quellen der Dichter annahm. Er gelangte zu diesem Schlufs in seiner Recension des Ogier von Voretzsch ( $\mathrm{LgrPh}$ I 895, Sp. 409), wo er folgendermafsen schrieb: „Für mich besteht kein Zweifel - aber ich hege nur geringe Hoffnung, mit meiner Ansicht ohne schweren Kampf durchzudringen - dafs der epische Ogier ... eine verhältnismäfsig junge Schöpfung ist. ... Der Dichter, der dieses Epos verfafste, entnahm seinen Stoff weder einem älteren Liede oder einer Reihe von Liedern, noch einer fertigen Sage, sondern er schuf seinen Heldentypus und dessen abenteuerliche Geschichte mit schöpferischer Dichterkraft. Des Dichters Quellen waren einerseits irgend welche karolingischen Annalen ..., anderseits das Sagenmaterial, das sich um den heiligen Othgerius von Meaux angesammelt hatte." Geschichtswerke und Legenden waren nach Becker auch die Quellen für die Wilhelmsepen: eine vorepische "Sage“ von Wilhelm dem Heiligen läugnet er geradezu (Wilhelm der Heilige S. 66 ff.). Freilich ist es kaum möglich, mit dieser Erklärung die Thatsache zu vereinigen, dafs in den Epen das Historische ebenso spärlich wie entstellt enthalten ist. So hat denn Becker in seinen neuesten Arbeiten diese These wesentlich eingeschränkt. Sie wird aber für die Geschichte unseres Problems stets von Interesse bleiben als eine Reaktion gegen die Form, in der die Lieder- und die Sagentheorie bisher wiederholt aufgetreten sind.

d) Eine vermittelnde und nicht dogmatisch verallgemeinernde Ansicht stammt von Gustav Gröber. Voretzsch bespricht sie S. 1 2-30. Auch nach Gröber sind vor den Epen kürzere Dichtungen entstanden, die „Zeitgedichte“. Diese wurden aber nicht etwa von den Epikern als Quellen benützt, sondern die beiden Gattungen blieben von einander unabhängig. Das älteste Zeitgedicht, von dem wir wissen, ist das sog. Farolied, das älteste Denkmal der Epik das Haager Fragment. Als ein Drittes kommt 


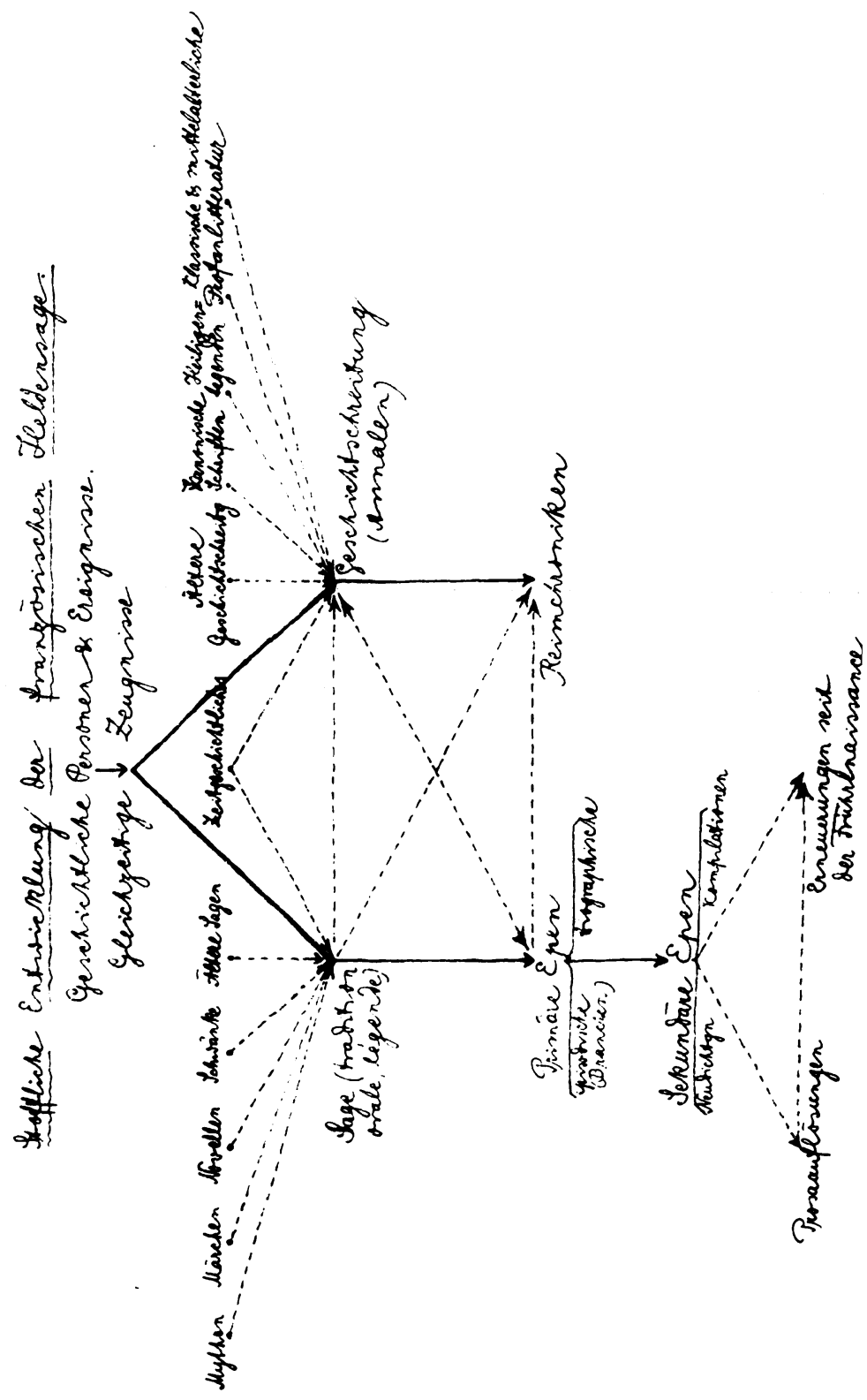




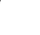

Brought to you by \| University of Ariz Authenticated

Download Date | 6/7/15 6:47 AM 
mündliche Ueberlieferung in Betracht, die sowohl von den Epikern als von den Chronisten als Quelle benützt worden ist. Voretzsch erklärt sich mit dieser Anschauung in der Hauptsache einverstanden, nur dafs er die für das Zeitgedicht vorgebrachten Belege anzweifelt und der Sage eine grö!sere Bedeutung zuerkennen möchte, als dies Gröber thut.

e) Als eine Weiterbildung der Gröberschen Gedanken stellt sich die Ansicht dar, die Fr. Ed. Schneegans neuerdings vorgeschlagen hat. Auch er erkennt die Volkssage nicht als hauptsächliche Quelle der Epen an. Um dies zu beweisen, nimmt er an, die ältesten Epen seien immer kurz nach den geschichtlichen Vorgängen entstanden, als eine annähernd treue Geschichtserzählung. Dagegen waren die Volkssagen von Anfang an reich an mythischen, märchenhaften und novellistischen Elementen. Dieser Unterschied beruhte darauf, da/s die Epen aristokratische Standespoësie, die Sagen der Besitz der untern sozialen Schichten gewesen seien. Erst als die Epen von oben nach unten ausgebreitet wurden, wurden auch sie mit den phantastischen Stoffen der Volkssage durchsetzt. - Was hier über den exklusiv aristokratischen Charakter der Epik gesagt wird, ist gewils richtig, das übrige aber zeigt sich als Hypothese, die den Thatsachen nicht entspricht. Voretzsch weist (S. 3I-47) nach, dafs schon die ältesten Epen, von denen wir wissen, von der geschichtlichen Wahrheit weit entfernt sind und der phantastischen Elemente so wenig wie die Sagen entbehren. -

So sehr die Meinungen über die Quellen der Ependichter heute auseinandergehen, scheint doch eine baldige Verständigung nicht ausgeschlossen. Voretzsch seinerseits ist bereit, auch andere Möglichkeiten im einzeinen Falle anzuerkennen. Eine einzige Ausnahme werfe eine sonst noch so einleuchtende Theorie über den Haufen. Wogegen er ankämpft, das sind die "aprioristischen" Lehren. Und man wird ihm hier kaum widersprechen können. Die Dichter pflegen ihre Stoffe zu nehmen, wo und wie sie dieselben finden. Theorieen von allgemeiner Beweiskraft für die Quellen irgend einer weitverzweigten Gattung lassen sich meines Erachtens überhaupt nicht aufstellen.

Ich habe hier die allgemeinen Richtlinien der Entwicklung der frz. Heldensage darzustellen versucht, so wie sie der Verfasser, wofern ich ihn recht verstanden habe, im Sinne hat. Zur Erläuterung sei nur bemerkt, dals die fetten Striche den häufigsten Weg der Entwicklung anzeigen, die unterbrochenen Striche andere als möglich nachgewiesene Beziehungen. Allgemein angenommen wird heute eine Scheidung der Epen nach sogenannten Stamm- oder Originalepen und abgeleiteten oder litterarischen Epen. Jene setzen keine ältere epische Bearbeitung ihres Stoffes voraus, diese aber sind erst nach dem Muster fertiger Werke desselben oder ähnlichen Inhalts geschaffen. Die Quellenfrage in unserem Sinne gilt daher überhaupt nur für die Dichtungen der ersten Art. Ich möchte 
die Namen „primäre" und "sekundäre" Epen vorschlagen, da diese Bezeichnungen durchaus unzweideutig sind. "Originalepen", $d . h$. Werke in der ursprünglichen Fassung, sind uns aus älterer Zeit überhaupt keine überliefert. „Stammepen“ drückt nicht den Gegensatz gegen die sekundären Epen aus, sondern nur den zu den später hinzugefügten Teilen eines Zyklus.

In die Sage werden erfahrungsgemäls allerlei phantastische Elemente aufgenommen, teils Altüberliefertes, teils Zeitgeschichtliches. Manches stammt aus heidnischen Mythen; diese Motive wurden aber schwerlich ihres religiösen Charakters wegen einverleibt, sondern um ihres ästhetischen Wertes willen. ${ }^{1}$ Die mythischen, märchenhaften, novellistischen, schwankhaften oder zeitgeschichtlichen Bestandteile machen manchmal das Ganze einer Sage aus, so dafs nur der Name des geschichtlichen Helden bleibt. Oft wurden auch ältere Sagen fertig auf jüngere historische Personen übertragen. Diese mannigfachen Quellen bleiben auch dem Geschichtschreiber nicht durchaus fremd; doch benützt er vorzugsweise schriftliche Quellen, ältere Geschichtswerke, kanonische Schriften, Heiligenlegenden und klassische oder mittelalterliche Profanlitteratur. Und allerlei Zeitgeschichtliches wird auch vom Historiographen verwendet.

Die primären Epen scheiden sich in zwei Gruppen: solche mit einfacher und solche mit mehrfacher Handlung (die sich technisch zu einander verhalten wie Novelle und Roman). Von der ersten Art sind z. B. einige von Voretzsch losgelöste „Branchen“ des Ogierepos, von der zweiten ist das Rolandslied. G. Paris hat die Artusromane, wo derselbe Unterschied vorliegt, in episodische und biographische eingeteilt. Dieselben Namen empfehlen sich vielleicht auch hier. Und ich möchte vermuten, dafs die episodischen Epen den mit der Sage vertrauten Hörern in Einem Stück vorgetragen, die gröfseren dagegen in Vortragsabschnitte zerlegt worden sind (darüber siche unten). Das wechselnde Bedürfnis scheint $\mathrm{zu}$ den beiden Gattungen geführt $\mathrm{zu}$ haben, die sich nur in der poetischen Technik unterschieden, jedenfalls von Beginn an neben einander existierten. Diese episodischen Epen sind übrigens, wie ich oben andeutete, die realen Ausgangspunkte der Liedertheorie gewesen.

Bei den sekundären Epen haben wir selbständige Neubearbeitungen zu trennen von blofsen Kompilationen, worin die übernommenen Werke nur äufserlich einander angepafst werden. Huon einerseits und Ogier andererseits sind von Voretzsch als Muster dieser beiden Arten aufgezeigt worden.

Zuletzt sind auf der Tabelle die Nachbildungen in jüngeren Zeitaltern eingezeichnet. Bojardo und Ariost, auch Victor Hugo wären hier als Beispiele anzuführen. Es sind die manchmal soge-

$1 \mathrm{Vgl}$. meinen Exkurs „Keltisshe Mythen in keltischer Heldensage und Legende", Gralsage S. $136-138$. 
nannten „Kunstepen“ im engeren Sinne des Worts, d. h. Erneuerungen innerhalb jüngerer Kulturverhältnisse, aus denen der Dichter seine Hörer mit Absicht in die früheren zurückversetzt.

2. Aufmerksamer als man es bisher that, hat Voretzsch die Komposition des Huon betrachtet. Er kommt, im Anschlufs an Sarans Ergebnisse in seiner Abhandlung über den Wigalois, zu dem bemerkenswerten Resultat (S. 151), dafs der Dichter des Huon die fünfteilige Kompositionstechnik der Artusromane sorgfältig nachgeahmt hat. Im alten Heldenepos herrschte das Nacheinander: eine Episode löste die andere ab, ob auch die Teile lose auseinanderfielen. Im ältesten Artusroman wird noch dieselbe Komposition geübt: so in den früheren Tristanromanen und im Lanzelet des Ulrich von Zatzikoven. Nach Saran (Paul und Braunes Beiträge XXI, S. 290 ff.) hat besonders Crestien die Technik des Nebeneinander ausgebildet: durch Einschachtelung wurden die einzelnen Handlungen zu einer unlösbaren Einheit zusammengefügt.

3. Nach Voretzsch hat der Huondichter in der Hauptsache zwei ältere Werke in einander verarbeitet, den "Urhuon" und den "Urhugo". Im ersteren, von dem uns ein Auszug in der Turiner Lothringerhs. erhalten ist, wurde die Mordthat eines Grofsen am Pariser Hof, seine Verbannung und Heirat mit einer ausländischen Königstochter erzählt. Das letztere Werk war eine ursprünglich fränkische Brautfahrtsage, deren Held mit Hülfe seines elbischen Vaters Alberich ein Ungeheuer erschlägt und eine Prinzessin zur Frau gewinnt. Diese fränkische Sage liegt andererseits auch dem deutschen Ortnit zu Grunde. Der Huondichter benützte sie in Form eines Epos. Es überrascht, dals G. Paris, in einem gleichzeitig erschienenen Aufsatz, 1 hier eine Sage annimmt. Voretzsch bemerkt darüber in einem Nachtrag: „Wir scheinen die Rollen getauscht zu haben".

Aufser diesen beiden Hauptquellen macht Voretzsch wahrscheinlich, dafs der Huondichter eine lange Reihe beliebter Heldenepen und ebenso die wichtigsten Artusromane gekannt und stofflich benützt habe. ${ }^{2}$ Hier wird der Leser wohl im Ganzen, nicht aber in allen Einzelfällen zustimmen. Wertvoll erscheint mir unter anderem der Nachweis, dals die Gestalt Auberons manche Züge aus den bretonischen Feensagen empfangen habe. In der That war zur Zeit des Huondichters in Frankreich die germanische Mythologie nicht mehr lebendig. $\mathrm{Da}$ war es nur natürlich, dafs man sich Alberich nach Art keltischer Mythenfiguren vorstellte. Ich hatte kürzlich die Vermutung geäufsert, dafs an Auberon vielleicht nur noch der Name germanisch sei. ${ }^{3}$ Uebrigens zeigt der französische Auberon merkwürdige Uebereinstimmungen mit Merlin.

Ueber den engin am Thor von Duonostre, die beiden Kupfer-

1 Romania 1900, S. 209-218.

2 S. 410 ist eine Filiationstabelle der benützten Werke beigegeben.

- Vollmöllers Jahresbericht IV, 2, S. 384 . 
männer, die beständig mit ihren Keulen losschlagen, giebt Voretzsch einen längeren Exkurs (S. 132-138). Er nimmt Benützung des Lancelot, Ivain und ursprünglichen Wigalois an. Dort findet sich aber nirgends das Motiv dieser Kupfermänner. Dagegen im Prosalancelot wird die Burg Dolereuse Guarde von drei Kupferriesen bewacht. Der erste steht auf dem inneren Thor und fält herab, sobald Lancelot die Mehrzahl der ihm unten entgegenstehenden zwanzig Ritter besiegt hat; doch trifft das Ungeheuer nicht Lancelot, sondern einen dieser Ritter. Hernach, als Lancelot, um die Bewohner der Burg zu erlösen, das letzte uud schwerste Abenteuer besteht und in die drei Gelasse eines tiefen Kellers eindringt, wird ihm vor der Thüre des zweiten Gelasses der Eintritt durch zwei beständig losschlagende Kupfermänner gewehrt; doch tritt er unverletzt ein. Im letzten Gelafs überreicht $\mathrm{ihm}$ eine kupferne Jungfrau einen Schlüssel.1 Ich will nicht behaupten, dafs diese Branche des Prosalancelot vom Huondichter benützt worden sei. Jedenfalls aber hat er die zwei schlagenden Kupfermänner aus keinem der von Voretzsch angezogenen Romane entnommen, sondern in irgend einem andern Artusroman vorgefunden. Ich erinnere mich, diesem Motiv noch öfter begegnet zu sein.

Voretzsch betont mit Grund, dafs das erhaltene Huonepos trotz der vielen stofflichen Entlehnungen nicht eine Kompilation, sondern eine gut komponierte Neuschöpfung sei (S. 53 ff.). Doch sind der inneren Widersprüche mehr als er anzunehmen geneigt ist. Als die lüsterne Esclarmonde. zu Huon in den Kerker tritt und ihre Wünsche sofort befriedigen möchte, weigert er sich aufs schroffste und wird erst durch mehrtägigen Hunger dahin gebracht, der Heidin die Ehe zu versprechen. Auf der Meerfahrt dagegen vollzieht Huon die Ehe mit der noch ungetauften Heidin, trotz Auberons strengem Verbot; Esclarmonde erinnert ihn umsonst daran, ringt flehend die Hände und rauft sich das Haar. Hernach in der Abtei unweit Bordeaux schläft Huon in einem andern Zimmer, um an diesem heiligen Ort nichts Unerlaubtes zu begehen (Guessard S. 175, 202, 270). - Ferner am Ende des Gedichts, nachdem er unter vielen Gefahren sein angestammtes Herzogtum Gascogne wieder erlangt hat, wird er von Auberon zum Nachfolger in seinem Feenreich ernannt und mufs versprechen, in drei Jahren dahin aufzubrechen. An diesen und andern Widersprüchen erkennen wir, dals der Huondichter das Thema des alten Huongedichtes verschoben hat. Der gläubige Heidenbekehrer des Heldenepos wird ihm zum Minnediener und abenteuerlustigen Ritter. Und dem Herzogtum, um dessen Besitz sich die Handlung des alten Epos gedreht hat, zieht Huon hier das fabelhafte Zauberreich im Orient vor. Voretzsch meint daher, eine "leitende Idee fehle ganz" und der Dichter habe nur die Absicht zu unterhalten (S. $73 \mathrm{ff}$.). Ich kann dieser Ansicht nicht beistimmen. Der Huondichter behält

1 Ich zitiere nach meinen Excerpten aus ff. 344: fol. $215^{\mathrm{d}}$ ff. und fol. $225^{\mathrm{c}} \mathrm{ff}$. 
wenigstens sein eigenes Thema fest im Auge und führt es sorgfaltig durch. Nur dals im Urhuon ein Thema verschiedener Art gegeben war. Beide haben sich dann gekreuzt, ohne dafs der Dichter und seine Hörer daran Anstols genommen hätten. Diese Themata will ich versuchen näher festzustellen. Vorher aber bleibt zu erwägen, an welches Publikum die Ependichter der älteren Zeit sich gewendet haben.

4. In Frankreich entwickelte sich eine Laienbildung zuerst an den Höfen des feudalen Adels, am Hof des Königs als höchsten Lehnsherrn wie an denen der unmittelbaren und mittelbaren Kronvassallen, der Herzöge, Grafen und Freiherrn. Die Feudalität, ${ }^{1}$ getragen von den ursprünglich fränkischen Adelsgeschlechtern, hatte sich im 10. Jh. zu solcher Macht erhoben, dafs die Kirche wirtschaftlich schwer geschädigt und auch als Kulturfaktor in ihrer beherrschenden Stellung eingeschränkt wurde. Neben den Bischofstädten und Abteien als den älteren Kulturzentren gedieh an den feudalen Höfen eine eigentümliche Bildung, die sich von der geistlichen mehr und mehr unabhängig machte. Courtoisie nannte man später diese älteste Laienbildung der neueren Zeit. Es war eine Weltanschauung und Lebensauffassung von ganz unkirchlicher und im Grunde auch unchristlicher Art. Laienrechtspflege und Kriegswesen bildeten die feste Grundlage. Auf dem Lehenrecht beruhte alle staatliche und gesellschaftliche Ordnung. Seine Kenntnis und Wahrung war die vornehmste Aufgabe der feudalen Gesellschaft. Durch beständige Kriege sicherte und erweiterte man seinen Besitz. Tapferkeit und Gewandtheit in der Führung der Waffen waren darum der zweite unveräufserliche Bestandteil feudaler Bildung. Das Lehenrecht war von Natur privatrechtlich, es betraf Beziehungen von Person zu Person. Auf persönliche Tüchtigkeit war demnach alles begründet, nur sie versprach Schutz und Sicherheit.

In den Kreisen dieses Feudaladels wurden die französischen Heldenepen zuerst gedichtet und vorgetragen. Es war eine ausschliefslich aristokratische, eine Feudalpoësie. Der Kirche war sie wenig genehm: umsonst versuchte man die Epen durch Legenden wie den Leodegar und Alexius zu verdrängen. Auch waren viele Bistümer und Abteien von Laien, Angehörigen der Feudalität, besetzt. Diese Kreise mögen an der Entstehung der Gattung wesentlich beteiligt gewesen sein. ${ }^{2}$ Erst später gelangte feudale Bildung und feudale Poësie auch in die Städte, auch hier zunächst nur zu den borgeois, den Patriziern. Kleinbürger und Bauern hatten im Zeitalter der Feudalität dringendere Sorgen, als ästhetische Genüsse zu pflegen. Die Vilains werden vom Ependichter überhaupt nicht erwähnt oder aber mit Schimpf

${ }^{1}$ Man pflegt statt von Feudalität vom Rittertum zu sprechen, trifft damit aber nur die militärische Grundlage der feudalen Gesellschaft.

2 Vgl. den kriegerischen Erzbischof Turpin im Rolandslied. 
and Spott bedacht.1 Der Hochadel und teilweise die hohe Geistlichkeit, hernach die Patrizier, dies war das Publikum, für das der Ependichter wirkte. Erst in der Zeit des Niedergangs, als andere poetische Gattungen an den Höfen in Mode kamen, trugen die Spielleute die „zersungenen" Epen auch ins niedere Volk.

5. Weltanschaung und Lebensauffassung des Feudaladels bildeten demgemäls das Thema des französischen Epos. Nach Lehenrecht werden alle menschlichen Verhältnisse beurteilt und geschildert. Die lehenrechtliche Terminologie wird der Grundstock der neuen Dichtersprache. Nicht sittliche, sondern rechtliche Konflikte sind es, die von den Epikern geschildert werden; ähnlich wie in der Ilias und im Nibelungenlied. Oftmals mifsverstehen wir eines dieser Epen, wenn wir von der Rechtsfrage absehen.

Ist das Recht verletzt, so können nur Krieg und Zweikampf die Ehre des Verletzten wiederherstellen. So sind Mord und Totschlag das häufigste Motiv der Ependichter geworden.

Und wie alle Rechtsordnung sich in persönliche Verpflichtungen auflöste, so finden wir in den Epen alles Geschehen aus Liebe und Hafs, Neigung und Widerwillen Einzelner erklärt. Durch diese seine Natur war das Lehenrecht weit entfernt, den Dichter vor undankbare Aufgaben zu stellen, im Gegenteil bot es ihm die reichste Fülle poetischer Konflikte.

In Wirklichkeit brachte, wie man weifs, das Zeitalter der Feudalität oftmals heillose Anarchie. Alles Recht war auf ein persönliches Treuverhältnis zwischen Senior und Vassall begründet. Darum war Treue die Kardinaltugend der feudalen Gesellschaft, Verrat $=$ Felonie das Kardinalverbrechen. ${ }^{2}$ Dichterische Gestaltungskraft konnte sich keine lohnenderen Motive wünschen. Und die feudalen Herren konnten und wollten nichts anderes lieber gepriesen hören als die Vassallentreue, und nichts so gebrandmarkt wie den Verrat am Herrn. Nicht blofs zur Unterhaltung hielten und belohnten sie den Dichter an ihrem Hofe. Der lehrhafte Zweck mochte manchem Fürsten das allein wesentliche sein, und dies umsomehr da die Bande persönlicher Treue im Leben so oft gebrochen wurden. Eine unverkennbare didaktische Tendenz wohnt der ganzen feudalen Epik inne. So materiell die Wirklichkeit, so ideell war die Dichtung.

Aus diesen Voraussetzungen begreift sich jede Zeile und jedes Wort des Rolandslieds. Auf der einen Seite verrät Ganelon aus Hafs gegen seinen Stiefsohn Roland und um Geld seine Mitvassallen und mittelbar den kaiserlichen Herrn an den Feind; auf der andern weigert sich Roland als Führer der Nachhut, als er schwer bedrängt ist, in sein Horn zu stofsen und dadurch das Hauptheer herbeizurufen, für dessen ungehinderten Abzug er Sorge zu tragen hat. Pur sun seignur deit hum suffrir destreis $e$ endurer

1 Vgl. die lesenswerte Studie von Josef Falk in den Mélanges de philologie romane dédiés à Carl Wahlund. Macon 1896, S. 109-122.

- Vgl. L, Gautier, Epopées I', S. 27. 
e grans cals e grans freis; sin deit hum perdre e del quir e del peil.1 Diese Worte Rolands sind das Motto des ganzen Gedichts. Und die Verurteilung und Hinrichtung des Verräters, deren ausführliche Schilderung uns heute befremdet, wurde von den damaligen Hörern mit besonderer Befriedigung vernommen.

Anders ist die rechtliche Lage des Kaisers in der Mehrzahl der Vassallenepen. Im Girart von Roussillon und Girart von Vienne - beide Dichtungen behandeln dasselbe Thema - gerät der Kaiser gegen seinen Vassallen dadurch ins Unrecht, dafs er sich mit einer Frau vermählt, die jenen liebt. Daraus folgt in beiden Werken grolses Unheil für Herrn und Vassall. Girart verliert dadurch ein ihm zugesichertes Land. Im Girart von Vienne wird der Konflikt noch verschärft. Als nämlich der Held zum Symbol der Huldigung des Kaisers Furs küssen will, veranlafst ihn die Kaiserin durch eine Täuschung, den ihrigen zu küssen, und rühmt sich hernach dieser Beschimpfung.

Im Raol von Cambrai beginnt der Konflikt damit, dals Ludwig dem nachgebornen Raol, Sohn des Grafen von Cambrai, seine angestammte Grafschaft entzieht, also die Erblichkeit des Lehens antastet. Später entzieht er, um Raol zu entschädigen, den vier Söhnen des Grafen Herbert von Vermandois ihr Lehen. Ein jahrelanger erbitterter Kampf um dieses Lehen ist die Folge. In dessen Verlauf verliert Raol das Leben.

Ein Rechtsstreit bildete auch das Thema des von Voretzsch erschlossenen Urhuon. Die zwei jungen Söhne des vor sieben Jahren verstorbenen Herzogs Sewin von Gascogne haben es durch Unkenntnis die ganze Zeit über versäumt, bei einem der Hoffeste Kaiser Karls zu huldigen, gleich ihrem Vater bei Tisch Vassallendienst zu leisten und sich im Besitz des kaiserlichen Lehens Gascogne bestätigen zu lassen. So hat Amauri, der selbst nach dem Königtum trachtet, leichtes Spiel, die pflichtvergessenen Vassallen bei Karl und dem soeben zum Thronfolger bestimmten Carlot der beabsichtigten Untreue $z u$ beschuldigen. Die beiden hätten ihr Lehen sofort verwirkt, wenn nicht Nales den Kaiser gebeten hätte, die beiden erst gütlich durch Gesandte an ihre Vassallenpflicht erinnern zu lassen. Es geschight, und dic Brüder erklären sich sofort bereit, dem Lehensherm durch den schuldigen Fufskufs zu huldigen. Auf dem Wege überfällt Carlot die Ungerüsteten, verwundet Gerart den jüngeren und fällt durch Huon, der ihn nicht erkennt. Die Brüder gelangen nach Paris. Huon erzählt den Ueberfall und wird vom Kaiser seines Schutzes versichert. Amauri bringt Carlots Leiche und behauptet, Huon habe diesen wissentlich getötet. Huon bestreitet es. Der gerichtliche Zweikampf entscheidet zu seinen Gunsten. Aber niemand hat das Geständnis des sterbenden Amauri gehört. Daher zweifelt Karl an der Richtigkeit des Gottesurteils und verbannt Hoon für immer

1 ed. Gautier v. 1010 und 1117 . 
aus Frankreich. Doch mildert er, auf das Einschreiten der Pers, dieses Urteil dahin ab, dafs Huon eine unmögliche Aufgabe erfüllen soll: komme er ohne die Beweise der vollbrachten That wieder, solle er ohne Prozefs gehängt werden. Der jüngere Bruder Gerart erhält das väterliche Lehen und verheiratet sich standesgemäls. F.r glaubt den Bruder tot und sich im festen Besitz der Herrschaft, als dieser nach der glücklichen Lösung der Aufgabe wiederkehrt. Fest entschlossen, auf das Herzogtum nicht freiwillig zu verzichten, weifs er dem arglosen Huon die Beweise der That $\mathrm{zu}$ rauben, setzt ihn gefangen, tötet seine Begleiter und erhebt beim Kaiser Klage, der Verbannte sei entgegen dem Verbot heimgekehrt. Huons Leben ist verwirkt. Er erbietet sich zum gerichtlichen Kampf gegen Gerart und dessen Schwiegervater Gibouart gemeinsam. Mit seinem Sieg über diese und deren Tod und Geständnis schlofs das alte Huonepos. Im erhaltenen Gedicht ist vom Dichter statt des Gottesurteils das zeitgeschichtliche Motiv der Perskammer eingesetzt. Huon hatte sich bereit erklärt, seinem Bruder die Hälfte des Herzogtums zu überlassen: dies scheint ein alter, charakteristischer Zug. Neu angefügt ist schliefslich Auberons Dazwischenkunft.

Dies war das Thema des alten feudalen Gedichts. Die Handlung bewegte sich darin um den Besitz des Herzogtums Gascogne, erst zwischen Karl und den Herzogssöhnen, dann unter diesen selbst. Um sein Erbe antreten zu können, vollbringt Huon das grolse Wagnis im Ausland; um es zu behalten, wagt Gerart Ehre und Leben. Einen Umschlag im Charakter des letzteren vermag ich nicht zu finden, wie Voretzsch (S. 75) annimmt. Dafs um ein Herzogtum auch dem Bruder die Treue gebrochen wird, konnte den damaligen Hörem nicht auffallen. Karl ferner tritt nicht, wie Voretzsch meint, „alles Recht mit Füfsen“, sondern ist formell in seinem Recht, wie auch die Pers anerkennen.

Dieses juristische Thema hat auch der Dichter des erhaltenen Huon beibehalten, ja sogar die Perskammer und ihre Sitzung noch hinzugefügt. Andererseits aber wollte er seinen Helden nach Art der modischen Minne- und Abenteuerromane schildern und prägte damit dem Ganzen ein neues Thema auf. Sein Werk gehört im Grunde nicht mehr zur Gattung der feudalen Epen, sondern zu den modernen Minneromanen. Den Höhepunkt der Handlung innerhalb des neuen Themas bildet die Verletzung des Keuschheitsgebots und der Seesturm. Die Liebenden werden getrennt und wahren einander auch in schwerer Gefahr die Treue. Esclarmonde schützt gegen einen aufgedrungenen Gatten ein Gelübde vor, und Huon verzichtet gern auf den Besitz der schönen Prinzessin, die sich im Schachspiel freiwillig von ihm hat schlagen lassen.

Das Mifsverhältnis des alten und des neuen Themas wird von den Hörern so wenig wie vom Dichter gefühlt worden sein. Dieses Erzeugnis der Spätzeit sollte weniger durch anregenden Gedankengehalt als durch bunte Fülle des Stoffs fesseln. Und dieses Ziel 
wurde am leichtesten erreicht, indem man zugleich den alten und den neuen Geschmack befriedigte.

6. Aelter als der Lehenverband war der Familienverband, die parente. 1 Er reicht noch in die germanische Zeit zurück. Ihm und seiner Macht ist es zuzuschreiben, dafs die Lehen, die ursprünglich mit dem Tode des Belehnten erloschen, früh erblich wurden. Die Familieninteressen konnten sich seitdem innerhalb der Feudalität mehr und mehr geltend machen. Diese Entwicklung zeigt sich besonders in den Vassallenepen, wie dem Lothringerepos, wo die Bordelois und Loherenc sich in Blutfehde gegenüberstehen. Ja die Ependichter versuchten, später fast sämtliche Personen einer Dichtung zu zwei feindlichen Geschlechtern zusammenzufassen. So läfst insbesondere der Huondichter seinen Helden überall mit Verwandten aller Art, Oheimen und Cousinen, unerwartet zusammentreffen: ein wesentliches Merkmal der jüngeren Epen, wie Voretzsch richtig bemerkt. Schliefslich versuchten die Urheber der grofsen Zyklen auch die Personen mehrerer Epen unter einander in Verwandtschaft zu bringen. Drei grofse Familien waren schliefslich das Ergebnis: die königliche, die der treuen und die der verräterischen Vassallen. Sogar in Artusromanen, im Prosatristan und Prosalancelot machte sich dieses Bestreben geltend. Es hatte seinen realen Grund in der überragenden politischen Bedeutung, welche einige grofse Fürstengeschlechter in Frankreich erlangt hatten.

7. Die älteren Epen zeigen meist eine eifrige Parteinahme für dieses oder jenes Fürstenhaus. Es lag in der Natur der Dinge, dafs der Ependichter, der an einem feudalen Hofe dichtete, dessen politisch-dynastische Interessen offen oder verhüllt vertrat. Vorliebe und Mifsgunst der einzelnen Hofdichter erscheinen stets so deutlich, dafs wir an der bestimmten Absicht nicht zweifeln können. Im Rolandsliede und andern Werken der sogenannten Königsgeste werden die Angehörigen des Karolingerhauses auf Kosten seiner Feinde verherrlicht. Hier werden die Pflichten des Vasallen gegen den höchsten Lehensherrn betont. Umgekehrt werden in den Vassallenepen dic Mitglieder der herzoglichen und gräflichen Familien als musterhafte Helden gepriesen und die Könige, der grofse Karl nicht ausgenommen, als schwach und haltlos, oft sogar als ungerecht und böswillig geschildert. Wir hören hier beständig von den Pflichten des Herrn gegen seine Vassallen und von Kränkungen der letzteren. So erkläre ich mir z. B. das Charakterbild Ludwigs im Kronungsepos. Wenn Kaiser Karl, und mehr noch sein Sohn Carlot, im Ogier und ähnlich im Huon so schlechtes Licht erhalten, möchte ich nicht mit Voretzsch (S. 75-76) irgend welche ästhetische Kritik üben. Es ist beidemal eine Invektive des Vassallendichters gegen das Königshaus. Mancher

1 Jacques Flach hat das Lehenwesen auf die parente zurückführen wollen, damit aber keine Zustimmung gefunden. 
schroffe Widerspruch mit der Geschichte, den man bisher dem "Walten der Sage“ zuzuschreiben geneigt war, dürfte sich auf diesem Wege aufhellen lassen. Die alte Einteilung in die drei Gesten findet hier eine innere Begründung. Wenigstens bei den älteren Epen wird uns die Frage cui bono? immer wesentlich fördern. Uebrigens schrieb A.W. Schlegel ${ }^{1}$ vor langen Jahren: „Wir sind so weit entfernt, alle Abweichungen der Sage blofs den Umwandlungen der blindlings wirkenden Zeit beizumessen, dafs wir vielmehr in nicht wenigen die absichtlichen Erfindungen einzelner Dichter sehen, welche dem Ahnenstolze dieses oder jenes Fürsten, oder seinen Ansprüchen auf erweiterte Herrschaft schmeicheln wollten. Wir glauben sogar die politischen $Z$ wecke zu erraten, zu deren Behuf manche Heldendichtungen, wo nicht zuerst ersonnen, so doch erneuert und in Umlauf gebracht worden sind."

8. Wir erhalten damit ein wertvolles Kriterium für Ort und $Z$ eit der Abfassung eines Epos. Die ursprünglichen Redaktionen der älteren Epen sind meines Wissens in keinem einzigen Fall sprachlich unverändert überliefert. So läfst sich hier aus sprachlichen und metrischen Kriterien nichts Sicheres gewinnen. Der F ürstenhof aber, nach dem die politisch-dynastischen Interessen einer Dichtung weisen, wird in jedem Falle als der Ort ernstlich in Frage zu ziehen sein, wo dieselbe gedichtet und zuerst vorgetragen wurde (unbeschadet ihrer späteren Verbreitung). Und da ergiebt sich uns, wenn wir die erhaltenen Werke überblicken, eine gewisse Anzahl feudaler Höfe, die wiederholt in Betracht kommen. Nächst Paris sind es vorzugsweise südfranzösische Fürstenhöfe (Narbonne, Orange, Bordeaux, Blaia (Blaivies), Vienne und andere. Was ferner die Abfassungszeit betrifft, so dürften sich öfter Uebereinstimmungen zeitgenössischer politischer Konstellationen mit den Situationen der Epen ergeben.

G. Gröber ist geneigt, manche Werke der Königsgeste, wie das Rolandslied, zeitlich möglichst nahe an die geschichtlichen Personen und Ereignisse zu rücken:2 nur zur Zeit der staallichen Blüte, ${ }^{3}$ nicht während des Niedergangs des Karolingerreichs können nach ihm die älteren Epen entstanden sein. Ich möchte die Richtigkeit dieser Begründung bezweifeln. ${ }^{4}$ Die Geschichte aller Völker lehrt uns, dafs die beschaulichen Künste, 5 so auch die Poësie, selten gleichzeitig mit grolsen staatlichen und kriegerischen Thaten gedeihen, vielmehr im besondern politische Dichtungen

1 ed. Böcking XII, 387 .

2 Frz. Literaturgeschichte S. 453.

[3 Bei mir steht von staatlicher Blüte nichts. Hrsg.]

[ + Die folgenden Ausführungen stimmen zu meiner Kenntnis der Lage Frankreichs in der Zeit vom 9.-I2. Jh. so wenig, dafs ich dem Verfasser in seiner Konstruktion nicht folgen kann. Hrsg.]

${ }^{5}$ Ich spreche auch nicht von beschaulichen Künsten und von Gleichzeitigkeit, sondern von gewissen Stimmungen des Volkes, die mir Voraussetzung für eine nationalpatriotische Heldendichtung zu sein scheinen. Hrsg.] 
diesen als ihr Nachklang folgen oder aber neuen Aufschwung vorbereiten. Gerade die Regierungszeit der französischen Karolinger und ältesten Kapetinger kann, wie mir scheint, Veranlassung gegeben haben, nach rückwärts zu blicken auf die ruhmreiche Vergangenheit und aus den Leistungen der Vorfahren Mut und Trost für die Gegenwart zu schöpfen. Frankreich seit Karl dem Kahlen bis vor Philipp Augusts zielbewulster und erfolgreicher Regierung war von der Machtfülle eines Pipin, Karl und Ludwig so weit entfernt, und stand gegen das kaiserliche Deutschland so sehr zurück, dals die dichterische Verherrlichung des weltbeherrschenden Kaiser Karl der Ausdruck politischer Wünsche und Hoffnungen genannt werden kann. In der Schlacht bei Bouvines mafsen sich zum ersten Male die Franzosen siegreich mit den kaiserlichen Deutschen. Der Traum begann Wirklichkeit zu werden. Kurz zuvor war die alte Karlssage für die malsgebenden Kreise zur blofsen Unterhaltungslitteratur geworden.

9. Die Frage nach der ursprünglichen Heimat der einzelnen Epen ist unlösbar verknüpft mit der alten Streitfrage, ob es aufser den wenigen überlieferten Epen in provenzalischer Sprache eine umfangreichere provenzalische Epik gegeben habe, ob z. B. die Wilhelmsepen zuerst provenzalisch abgefafst gewesen seien. Eine grofse Anzahl gerade der wertvollsten Dichtungen würden dann dem Süden angehören. Für die Beantwortung dieses Problems hat uns Franz Saran kürzlich einen beachtenswerten Fingerzeig gegeben.1 Er schlägt vor, in das Mittelalter den Begriff der "Gattungssprache" einzuführen. So seien in Griechenland für die einzelnen poetischen Gattungen bestimmte Mundarten üblich geworden, nachdem hervorragende Dichter diese Mundarten zu Kunstsprachen für bestimmte Gattungen ausgebildet hatten. Es ist eine bekannte Thatsache, dals der attische Tragiker dorische Chorlieder dichtete und in seine Tragödien einlegte. Die Mischsprache Homers war noch im alexandrinischen Zeitalter die Sprache des Epos schlechthin. Eine solche poetische Gattungssprache gab es im Mittelalter auf der Pyrenäenhalbinsel. Alfonso X. von Spanien beteiligte sich eifrig an der kastilianischen Litteratur; nur für seine Lyrik, die weltliche wie die geistliche, bediente er sich des GalizischPortugiesischen, das von einer zahlreichen Dichterschule für diese Gattung ausgebildet worden war.

Sollte Aehnliches auch in Frankreich der Fall gewesen sein? Die bekannte Bemerkung des Ramon Vidal, das Französische sei für Romane und Pastourellen, das Provenzalische für Minnelieder und Sirventese besser geeignet, scheint solche Verhältnisse vorauszusetzen. Vom Provenzalischen jedenfalls steht es fest, dafs es für Minnesinger aus italischem, katalanischem und auch nordfranzösischem Sprachgebiet die anfangs allein mögliche Gattungssprache gewesen ist. Schon Wilhelm IX. von Poitou, der älteste Trouba-

1 Germanischer Jahresbericht 1899, S. 79. 
dour, dessen Werke uns zu einem Teil überliefert sind, bediente sich nicht des Poitevinischen oder einer andern nördlichen Mundart, sondern der bereits damals ausgebildeten provenzalischen Dichtersprache auf limousinischer Grundlage. Andererseits war das Französische die Gattungssprache des Epos. Wir sehen dies besonders an den französischen Epen in Oberitalien. Ob auch südfranzösische Dichter sich im Epos des Französischen bedient haben, bliebe noch zu untersuchen. Die Existenz so vieler französischer Heldenepen, worin südliche Helden und südliche Herrscherfamilien gefeiert werden, wäre damit kein Problem mehr.

Heldenepos und Minnesang waren höfische ${ }^{1}$ Gattungen. Das gebildete Publikum, für welches dieselben bestimmt waren, mochte ohnedies beide Kultursprachen Frankreichs beherrschen. So boten sich dem sprachlichen Verständnis keinerlei Schwierigkeiten. Die französischen Epen, gleichviel wo entstanden, waren im Norden und Süden gleich willkommen. Von der Beliebtheit der Epik im Süden zeugen uns die zahlreichen Anspielungen der Troubadours, auf die man längst aufmerksam geworden ist. Umgekehrt wurden provenzalische Minnesinger an nordfranzösischen und dem englischen Hofe aufgenommen, und besangen dort die Fürstinnen in provenzalischer Sprache. Erst im letzten Drittel des 12. Jhs. begann man den Minnesang in französischer Sprache zu pflegen. Und im 13. Jh., als sich infolge der Albigenserkriege das Uebergewicht des Nordens über den Süden mehr und mehr geltend machte, wurde die provenzalische Kultursprache allmählig zurückgedrängt.

Damit wird uns ein merkwürdiger Zusammenhang $\mathbf{z w i s c h e n}$ Heldenepos und Minnesang klar. Es mufs auffallen, dafs dieselben Kulturzentren die Heimat wertvoller Epen und hervorragender Minnelieder gewesen sind. In Narbonne, dem Brennpunkt der Aimeri-Epen, wurde die Vizgräfin Ermengard von Peter Rogier und andern Troubadours besungen. In Blaia, von dessen Grafenhaus die Geste de Blaivies handelt, ${ }^{2}$ lebte Jaufré Rudel, der Held der schönsten Troubadournovelle. Graf Raïmbaut von Orange stammte aus derselben Grafschaft, deren Namen Wilhelm der Heilige trug. Auch 'Toulouse, Bordeaux, Vienne und andere Höfe wären hier zu nennen, nach denen sowohl frz. Epen wie provenzalische Lieder weisen.

Aber auch ein innerer Zusammenhang zwischen Heldenepos und Minnesang wird sich uns ergeben, wenn wir erwägen, dafs diesen beiden Gattungen feudaler Hofpoësie dieselbe Tendenz gemeinsam ist. Wie immer man sonst über das Wesen des Minne-

1 Man bat sich gewöhnt, Minnesang und Minneroman als „höfische Dichtung" dem sogenannten „Volksepos" gegenüberzustellen. Dieses aber ist feudale Hofpoësie nicht minder als jene beiden Gattungen. Besser würde man thun, dieselben als Minnepoësie zu bezeichnen, da der Frauendienst ihr gemeinsames Merkmal ausmacht. Vgl. meine Gralsage S. 52 ff.

2 Vgl. G. Paris, Revue historique LIII, 1893, S. 226 Anm. 
sangs denken mag, eine panegyrische und politisch-dynastische Spitze läfst sich dieser Verehrung fürstlicher Damen kaum absprechen. Vertrat der Sirventesdichter in politischen Liedern die Sache seines Herrn, so feierte der Minnesinger die Vorzüge der Herrin. Meist vereinigte der Troubadour in sich diese doppelte Aufgabe. Ja, einige der bedeutendsten wagten es sogar, beide Themen in demselben Liede $z u$ verbinden.

Heldenepos und Minnesang unterschieden sich, wie mir scheint, vor allem darin, dals das Epos mit seinem kriegerisch-politischen Inhalt vorzugsweise für Männer, das Minnelied mit seiner Liebespsychologie zunächst für feingebildete Frauen bestimmt war. In Südfrankreich, wo das römische Erbrecht nicht durch das germanische verdrängt worden war, konnten Frauen das ererbte Lehen antreten und selbständig regieren. So war hier die rechtliche Stellung der weiblichen Angehörigen des Hochadels wesentlich anders als im Norden. Damit stimmt merkwürdig überein die verschiedene Auffassung und Würdigung der Frau im Epos einerseits und im Minnelied andererseits. Doch darf nicht unerwähnt bleiben, dafs die aus dem Süden stammenden Epen hierin dem Minnesang näher stehen. Im alten Rolandslied, das sicher im Norden verfarst ist, tritt überhaupt keine Frau innerhalb der Handlung hervor; Rolands Braut Alda ist erst später aus einem andern Epos eingeschoben worden. Dagegen sind Elissent und Bertha im Girart von Roussillon, und ähnlich Guibourc in Aliscans mit sichtlicher Vorliebe geschildert. Insbesondere in Girarts Verhältnis zu seiner Herrin und früheren Braut, der Kaiserin Elissent, glaube ich eine Art Vorahnung des späteren Frauendienstes zu finden.

Sollte sich diese Annahme einer epischen und einer lyrischen Gattungssprache Frankreichs bei näherer Prüfung bewähren, so stünden altfranzösische und altprovenzalische Litteratur nicht mehr in scharfer Trennung neben einander. Wie Frankreichs mittelalterliche Kultur überhaupt, würde sich uns auch seine Poësie als geschichtliche Einheit erweisen.1 Und die Gelehrten hätten Recht, welche sich gewöhnt haben, die Geschichte der französischen und der provenzalischen Litteratur im Zusammenhang $\mathrm{zu}$ behandeln.

10. Wer aber waren die Verfasser der älteren Heldenepen, von Roland, lsembart und Gormund, Krönungsepos, Aliscans? Oder, um die Frage genauer zu formulieren, was war ihr Bildungsstand und wie ihre gesellschaftliche Stellung?

Haben wir uns, mit Gröber,2 Krieger als die Verfasser zu

${ }^{1}$ Die drei hauptsächlichen poëtischen Gattungen im mittelalterlichen Frankreich, die rings in die Nachbarländer hinausdrangen, sind Heldenepos, Minnesang und Minneroman. Nordfrankreich ist die Heimat der ersten Gattung, der Süden die der zweiten; und wieder im Norden entstand aus einer Uebertragung der Minne auf die feudalen Ritter die dritte. Ich habe diese Entwicklung darzulegen versucht in meiner Gralsage S. 49-53. Hrsg.]

${ }^{2}$ Frz. Litgesch. S. 456. [Bei mir steht von "einfachen Soldaten" nichts. 
denken, einfache Soldaten, die als Augenzeugen das Miterlebte schilderten, gleich Werimbert, dem Gewährsmann des Mönchs von St. Gallen? So wären es also Dilettanten, nicht Berufsdichter gewesen. Aber vermochten solche die hochentwickelte Technik, die uns schon in den ältesten Werken begegnet, ohne weiteres zu handhaben oder gar zu schaffen? Die künstlerischen Kenntnisse, die zur Abfassung auch eines mittelmälsigen Epos nötig waren, können nur durch berufsmälsige Tradition und schulmälsige Mitteilung ausgebildet und bewahrt worden sein. Diesen Kriegsleuten, welche Gröber ${ }^{1}$ im Sinne hat, möchte ich, mit Voretzsch, nicht die Schaffung von Epen, wohl aber die Pflege der Sage vorzugsweise zuschreiben.

Oder haben wir uns unter den Verfassern die vielbesprochenen Spielleute zu denken, histriones - joglëors, Leute, die vom Vortrage von Dichtung und Musik und allerlei Schnurrpfeifereien lebten und wandernd die Höfe, Städte und das platte Land durchzogen? Dem widerspricht die durchaus ernsthafte, auf Recht und Sitte gerichtete Lebensauffassung, mit der die Dichter in älterer Zeit ihrem Thema gegenüberstehen. Dem widerspricht die feierliche Würde des Vortrags, das freimütige, oft kühne Urteil über hochstehende Personen, die sichere Beherrschung der standesgemälsen Lebensformen, und nicht zuletzt der feine künstlerische Geschmack. Die Ependichter haben wir unter den Gebildetsten ihrer Zeit, unter den geistigen Wortführern zu suchen, nicht aber unter fahrenden Leuten und armen „Tellerleckern". Der Bildungsstand und die gesellschaftliche Stellung, die sich in den Epen der besseren Dichter offenbaren, weisen nicht auf die Strafse, sondern auf die kulturellen Brennpunkte.

Sollen wir daher mit L. Gautier ${ }^{2}$ kurzweg clercs als die Urheber der Heldenepen vermuten, Dichter wie die des Leodegar und des Alexius? Gerade diese Beispiele Gautiers zeigen uns deutlich den unüberbrückbaren Gegensatz zwischen kirchlichen Legenden und feudalen Epen. Dort wird die kirchliche Weltanschauung, und als ihr Hauptteil die Askese gepredigt. Hier dagegen schäumt der Wille zum Leben, weltliche Thatkraft und Thatendrang. Und, wenn ich nicht irre, wollte die Kirche gerade die Weltlust, die hier gedeiht, durch ihre Legendenlitteratur bekämpfen. Von kirchlichem Leben und aufrichtiger Weltentsagung

[ ${ }^{1}$ Bei mir heifst es 1. c.: „Also in den Reihen von Kriegern ... wird das frz. Heldengedicht seinen Ursprung gehabt haben und grofs geworden sein". Und Herrigs Arch. l. c. S. 32I: "Und da für das geringste litter.rische Erzeugnis, auch für das sog. Volkslied, ein wenn auch noch so geringer Grad sprachlichen Bewurstseins und litterarischen Verstandes erforderlich ist, werden diese Eigenschaften auch den ersten dichtenden Bewunderern Karls d. Gr.... (unter den Franzosen) ... nicht abgesprochen werden können". Dals es dichterisch befähigte Krieger unter den Kämpfern der Karolingerzeit gab, zeigt uns das rhythmische lat. Zeitgedicht im Volkston des Kriegers Angilbert auf die Schlacht von Fontenoy vom Jahre 84 I (s. Grundrifs II I, 168). Hrsg.]

${ }^{2}$ Épopées II', S. 40-45. 
vermag ich in der französischen Epik wenig zu finden. In den verschiedenen Moniages sehe ich im Gegenteil einen Protest gegen die mönchische Zumutung, sich einen Helden wie Wilhelm in der Klause eingesperrt zu denken.

Wohl aber besalsen die Ependichter ohne Zweifel geistliche Bildung: in diesem Sinn also waren sie clerc. ${ }^{1}$ Sie hatten sich die höchste mögliche Bildungsstufe erworben, die eben nur von der Kirche bezogen werden konnte; oder, in die heutige Sprache übersetzt, sie besalsen „akademische Bildung".2 So erkläre ich mir den ausgesprochen christlichen Charakter der frz. Epik und die z. B. im Rolandslied unläugbare Benutzung heiliger Schriften, wie der Bibel insbesondere. Ein clerc war überdies jener Turoldus, der am Rolandslied als Redaktor oder Verfasser beteiligt ist. ${ }^{3}$ Clerc nennt sich ausdrücklich Bertrant von Bar sur Aube. Suchier bemerkt dazu: „Dafs er ein Kleriker gewesen ist, würde ohne seine bestimmte Angabe niemand vermuten".4 Auch der Verfasser des Lothringerepos befals nach F. Lot "une certaine instruction".5

Aber die frz. Epiker waren weit entfernt, ihre geistliche Bildung in den Dienst der Kirche zu stellen und etwa Legenden wie den Alexius zu verfassen. Vielmehr wurzelten sie mit all ihren Anschauungen in dem Leben der feudalen Höfe, wo Rechtspflege, Krieg und Jagd das Ziel und die Aufgaben des Mannes darstellten, und christlicher Glaubenseifer sich ausschliefslich in den Fehden mit den Mohamedanern Spaniens und den germanischen Wikingern bethätigte. In diesem Kreise, als Mitglieder der maisniée, müssen sie gelebt und gedichtet haben. Grofsenteils auch mögen sie, wie Bertrant, dem Adel von Geburt angehört haben, wenigstens dem niedern Adel, als Söhne eines Kastellans oder Vavassors. Oder aber errangen sie sich ähnlich einem Bernhard von Ventadorn trotz unfreier Abkunft durch Begabung und Leistungen die Hoffähigkeit. Sie safsen mit zu Gericht im Rate der Vassallen, und zogen mit dem Fürsten zur Fehde und auf die Jagd. So nennt sich Raimbert von Paris, Redaktor des Ogierepos, einen Edelmann.6 So wird auch Taillefer, der bei Hastings dem Normannenheer voranritt und aus dem Rolandsliede sang, noble vassal genannt.?

Jetzt erinnern wir uns ähnlicher Gestalten aus dem deutschen Heldenepos, Volkers im Nibelungenlied und Horands in der Gudrun. Damit werden wir unmittelbar auf das gemeingermanische Amt des skop zurückgeführt, des hochangesehenen Hofdichters, den

1 Hertz, Spielmannsbuch ${ }^{2}$ S. 4.

2 Auch Fr. Kauffmann erschliefst für das Hildebrandslied einen vornehmen und geistlich gebildeten Verfasser. Sieversband S. 178.

${ }^{3}$ Suchier, Frz. Litgesch. S. 25.

1 Frz. Litgesch. S. 28.55.

5 Rom. XXVIII, 1899, S. 279. Vergl, auch Aimeri ed. Demaison S. LXXVIII.

- Gautier, Epopées II², S. 46.

'Freymond, Jongleurs et Ménestrels S. I3-I4. 
schon Hertz ${ }^{1}$ als Vorläufer der mittelalterlichen Dichter bezeichnet hat. Das frz. Epos wird heute allgemein als christliche Fortsetzung verlorener fränkischer Epen aufgefalst. So trat auch der frz. Epiker an die Stelle des fränkischen skop. Nur dafs er sich von diesem durch seine christliche Schulbildung unterschied. Seine soziale Stellung war dieselbe. Ein besonderer Name scheint für ihn nicht üblich geworden $\mathrm{zu}$ sein. Vermutlich nannte man ihn kurzweg clerc, oder aber begriff man ihn unter die übrigen Vassallen am Hofe mit ein? (Vielleicht auch hiefs er chantëor?) So konnte es geschehen, dafs ihn die Kirche mit dem Namen der litterarischen Zwischenhändler, der histriones, bezeichnete. Erst in Südfrankreich und zunächst nur für den Minnesänger kam eine Bezeichnung auf, durch die auch äufserlich der Dichter vom Spielmann geschieden wurde: es war der Name trobador, der früh nach dem Norden kam und dort weitere Ausdehnung erhalten zu haben scheint.

Nach Bildung, Lebensstellung und Leistungen weitab von den Epikern der feudalen Hofgesellschaft standen die Spielleute. Sie lebten vom Vortrag und der Verbreitung dieser Epen und anderer Dichtungen. Als $Z$ wischenhändler übernahmen sie die Aufgabe, die heute dem Buchdrucker und Buchhändler zukommt. Zwei Gruppen lassen sich unter ihnen erkennen, die aber nicht scharf geschieden waren. Manche waren an einem Fürstenhofe dauernd angestellt; sie hiefsen menestrel, Bedienstete. Diese bethätigten sich teilweise auch als Dichter, suchten es also den andern nachzuthun. Sie bedienten sich dabei der überlieferten Technik und der zahlreich vorhandenen Muster. Bekannte poetische Vertreter dieses Standes sind Estrumen, der menestrel cortois des Admirals Gaudisse, und Pinchonnet im Cléomades, auch Daurel in Daurel und Beton. Die berühmtesten menestrel, von denen wir wissen, sind Jean Bodel aus Arras und Baudouin und Jean aus Condé, ferner Adenet der Spielmannskönig. Auch der Huondichter gehört zu ihnen: er hat im Estrumen ein Bild seines Standes gezeichnet, wobei er freilich weniger der Wirklichkeit als seinen Wünschen gefolgt sein wird. Aus dem Nibelungenlied sind Werbel und Swemmel, die Hofspielleute König Etzels, hier zu nennen. Diese menestrel pflegten die Poësie, soweit sie sich daran beteiligten, mehr nur des Gelderwerbs wegen und als Unterhaltungslitteratur. Um hohe didaktische Ziele oder die kluge Vertretung politischdynastischer Zwecke war es ihnen kaum mehr zu thun. Sie verhielten sich zu den Nachfolgern des skop wie heute der Journalist zum Dichter.

Gesellschaftlich tiefer als der Hofspielmann stand der Fahrende, joglëor schlechthin. Er mulste seinen Unterhalt suchen, wie er ihn fand, und wenn nötig auch das Gewerbe des Possenreilsers ausüben. Selten trat er als Dichter auf; wenn es geschah, travestierend oder parodierend. Derbe Komik war sein liebstes Thema.

1 Spielmannsbuch ${ }^{2}$ S. 2. Dort weitere Litteratur. 
Auch der menestrel mufste, wenn sein Herr starb oder ihn entliefs, sein Brot anderwärts suchen. Der Estrumen im Huon gerät in diese Lage. Eine feste Grenze zwischen dem ansässigen und dem fahrenden Spielmann bestand nicht. So wurden denn auch die Namen bald promiscue gebraucht. Auch diesen wechselnden Sprachgebrauch beobachten wir im Huon.

Sobald ein Epos in die Hände der Spielleute geraten war, konnten Entstellungen im mündlichen Vortrag nicht ausbleiben. Und als an den Feudalhöfen Minnesang und Minneroman in Mode kamen und die alte Epik allmählig aus den malsgebenden Adelskreisen verdrängt wurde, war der Verderbnis Thür und Thor geöffnet. Erst wurden die stolzen Werke den Patriziern der Städte, dann den Kleinbürgern und schliefslich den vilains jeder Art ausgeliefert. Wir können an manchem Epos verfolgen, wie es „zersungen", d. h. erweitert oder verstümmelt wurde. Für den gedanklichen Gehalt war in den unteren Schichten keinerlei Verständnis mehr, ebenso wenig für die künstlerischen Ausdrucksmittel. Das rein stoffliche Interesse blieb übrig. Je mehr das Adelsepos „Volksepos" wurde, Gemeingut der niederen Volkskreise, desto mehr wurde es entstellt. Am Anfang hatten grofse dichterische Persönlichkeiten gestanden, am Ende nahm die Menge das wertvolle Gut in Besitz. Von oben nach unten, von engen Zentren ins Weite ging der Weg, den die Heldenepen nahmen. Es ist der erfahrungsmäfsige Gang aller Kulturentwicklung.

II. Wann aber traten französische Hofdichter an Stelle der fränkischen skopas? Mit andern Worten, in welche Zeit ist der Anfang des französischen Heldenepos zu setzen? Wie wir sahen, war dieses nach Wesen und Entstehung höfische Dichtung. So können wir um den Zeitpunkt nicht verlegen sein. Erst als das Galloromanische anstatt des Fränkischen Hofsprache geworden war, kann ein romanisches Heldenepos gedichtet worden sein. Wann die fränkischen Eroberer ihre germanische Sprache aufgegeben haben, darüber fehlen meines Wissens eingehende Untersuchungen. Karl der Grofse und sein Hof sprachen als Austrasier Fränkisch; und wohl noch Ludwig der Fromme. Die Strafsburger Eide 842 zeigen die neustrischen Franken bereits romanisiert. Nach allgemeiner Ansicht vollzog sich dieser durch Zweisprachigkeit der Franken vorbereitete Sprachentausch unter Karl dern Kahlen. Mit der Trennung von den ausschliefslich germanischen Landesteilen wurde das Galloromanische allgemeine Landes-, auch Hofsprache; ähnlich wie seit den Ereignissen des Jahres 1866 das Deutschtum in Oesterreich-Ungarn in seinem Besitzstand bedroht wird. Ich treffe also mit Hermann Suchier zusammen, der den Beginn des frz. Heldenepos erst ins neunte Jh. verlegt, nicht aber ins achte oder gar ins sechste, wie andere Gelehrte wollen.

12. Warum hat sich auf deutschem Boden kein Epos von den Karolingern entwickelt? Diese Frage, obzwar sie nur akade- 
mischer Art ist, fordert immer wieder zur Prüfung heraus. ${ }^{1}$ Einige Karlssagen und vielleicht auch Gedichte waren vorhanden. Der bekannte Mönch von St. Gallen und einige andere Zeugnisse berechtigen uns zu dieser Annahme. ${ }^{2}$ Es bestand jedenfalls einmal ein Gedicht in althochdeutscher Sprache über Karls Anrücken gegen das von Desiderius und Otker verteidigte Pavia. Auch Voretzsch räumt das ein.3 Aber eine eigentliche Kpik von Karl oder überhaupt aus diesem Zeitraum, etwa über Widukind, von dem französische Dichter so viel erzählten, kam in Deutschland nicht zur Reife. Hier beschränkte sich das epische Gut auch noch später auf eine Heldensage und Heldendichtung aus dem Zeitalter der Völkerwanderung. Walther und Hildegund, Hagen und Siegfried, Dietrich von Bern und der Hunnenkönig Etzel blieben die Helden auch der deutschen Epik des 12. Jahrhunderts.

Wenn die Kämpfe im Zeitalter der Karolinger zu einem deutschen Heldenepos nicht geführt haben, so möchte ich den Grund nicht etwa in der politischen Gegnerschaft der Alemannen, Baiern und Sachsen gegen Karl den Grolsen erblicken. Wie Etzel hätte der Kaiser Karl auch als Feind ins deutsche Epos gelangen können. Mir scheint die Ursache vielmehr in den allgemeinen Bildungsverhältnissen Deutschlands zu liegen. In der ahd. Sprachperiode - das zeigt uns besonders deutlich Kelles Literaturgeschichte - gab es in Deutschland thatsächlich nur eine lateinisch-kirchliche Bildung und Litteratur. Noch fehlte eine von der Kirche unabhängige Laienbildung, es fehlte eine deutsche Kunstund Kultursprache. Ja die Kirche beherrschte das geistige Leben so unumschränkt, dafs sogar die vorhandenen alten Epen, die Werke der alten skopas aus dem Zeitalter der Völkerwanderung von den mafsgebenden Bildungsstätten ausgeschlossen und einigen abseits liegenden Höfen überlassen blieben. Dort, in der Hand ungebildeter Dichter, wurden jene Epen vielfach entstellt und verwirrt. Nur vereinzelt taucht diese poëtische Gattung in der lateinischen Litteratur auf: es ist der Waltharius manufortis und das uns bezeugte lateinische Nibelungengedicht.

Erst im Laufe des I 2. Jh. gelangte die Laienbildung der französischen Feudalität auch nach Deutschland herüber. Jetzt erst machten sich die Hofkreise von der kirchlichen Bevormundung frei. Aber als ein deutscher Fürst das Rolandslied übertragen liefs, da arbeitete der Uebersetzer das feudale Epos sorgfältig im kirchlichen Sinne um. Und nur langsam wich die Kirche aus ihrer beherrschenden Stellung. Erst unter Friedrich Barbarossa brach französische Bildung und Gesittung unaufhaltsam herein. Jetzt erst war auch für die einheimischen Epen die Zeit der Wiederherstellung gekommen. Aber an den höfisch verjüngten Werken blieben

1 Gröber, Frz. Litgesch. S. 456.

2 Bernheim, Preufs. Jahrbücher LXXXI (1895, 3. Bd.) S. 345-358.

3 Ogier S. 29. 
die Spuren langer Vernachlässigung untilgbar haften. In den weitaus meisten Fällen zogen es statt dessen die deutschen Hofkreise vor, sich fertige Epen und Romane aus Frankreich übertragen zu lassen.

13. Die Epen wurden, wenn sie gröfseren Umfang hatten, in Vortragsabschnitte geteilt und abschnittweise vorgetragen.1 In mehreren Werken, so im Huon (Guessard S. 33, 148 und 164) werden die Pausen ausdrücklich angemerkt. Mit der Disposition des Ganzen brauchten sich diese Rhapsodieen nicht zu decken. Im Huon wenigstens scheinen die Einschnitte absichtlich mitten in die Erzählung verlegt worden zu sein. So wurde die Spannung der Hörer besser wach erhalten.

Aus der Gewohnheit dieser Einteilung für den Vortrag erklärt sich, wie mir scheint, ein eigentümlicher Stilgebrauch des Heldenepos. Voretzsch (S. 77) macht darauf aufmerksam, dafs im Huon oft eine Erzählung mit denselben oder ähnlichen Worten wiederholt werde, so der Ueberfall und Tod des Carlot. Aber diese Wiederholung, die nicht weniger als drei Textseiten einnimmt, befindet sich unmittelbar nach dem ersten Vortragsabschnitt (Guessard S. 33). Der Dichter fand es geraten, zu Beginn der zweiten Rhapsodie, nachdem er die Hörer um Ruhe gebeten hatte, das entscheidende Ereignis, mit dessen Erzählung er Tags zuvor abgeschlossen hatte, nochmals und zwar durch Huon selber vortragen zu lassen. Aehnliche Fälle finden sich öfter. Dieser Brauch der Wiederholungen ergab sich aus der Art und Weise des Vortrags.

Auf diese knappen Bemerkungen will ich mich an dieser Stelle beschränken. Sie wurden, wie ich oben vorausschickte, veranlafst und angeregt durch die von Carl Voretzsch begonnenen „Epischen Studien", als eine Sammlung von Vorarbeiten zu einer Geschichte der französischen Heldensage. Meine Absicht war, das was ich hier darzulegen versucht habe, zur Diskussion zu stellen. Später denke ich einen oder den andern Punkt ausführlicher zu begründen.

1 Ueber Vortragsabschnitte bei Crestien vgl, meine Gralsage S. 159-161.

EDUARD Wechssler. 